and family carers play an important role in managing pain medication at home. There is evidence carers need support with this, yet no research in the UK has developed and tested theorydriven interventions to help them manage pain medicines. We are conducting a Phase I-II feasibility study (as defined by the Medical Research Council guidelines for developing a complex intervention) to develop a new intervention: Cancer Carer Medicines Management (CCMM).

Aim To summarise the development of CCMM, outlining the process of intervention development.

Method A staged multi-method approach was used to obtain current evidence to inform CCMM's development: a systematic literature review of interventions for carer end of life medicines management; a scoping exercise concerning practice and theoretical contexts; interviews and user involvement group consultations with patients, carers, and health professionals with palliative care experience. This information informed two workshops which used participatory action research processes to design and develop an intervention which will be further refined through a consultative review process.

Results To date we have found that this staged approach is appropriate and successful in enabling an evidence based intervention to be designed collaboratively. This also provides a means by which the clinical applicability of the intervention can be refined as it develops.

Conclusions Refining this intervention as it develops will ensure that it reaches the feasibility trial stage with a robust grounding in carer experience, clinical practice and research evidence. In turn, the intervention will have the potential to enhance the practice of nurses in the community to meet carers' needs in pain medication management. This project is funded by the Dimbleby Marie Curie Cancer Care Research Fund; grant number DCMD-RF-12-05.

OP 018 CANCER CARERS MEDICINES MANAGEMENT:

\title{
A FEASIBILITY TRIAL OF AN EDUCATIONAL INTERVENTION FOR MANAGING END OF LIFE PAIN MEDICATION
}

Jane Hopkinson, ${ }^{1}$ Jacki Hughes, ${ }^{1}$ Elizabeth Lowson, ${ }^{2}$ Alison Richardson, ${ }^{2}$ Sue Duke, ${ }^{2}$ Sally Anstey, ${ }^{1}$ Mike Bennett, ${ }^{3}$ Peter Smith, ${ }^{2}$ Carl May, ${ }^{2}$ Sue Latter ${ }^{3} .{ }^{1}$ Cardiff University, Cardiff, Wales, UK; ${ }^{2}$ University of Southampton, Southampton, UKi

${ }^{3}$ University of Leeds, Leeds, UK

10.1136/bmjspcare-2014-000654.18

Background Cancer accounts for 1 in 4 deaths in the UK. A majority of people with advanced cancer will experience pain 